\title{
Detection of mild cognitive impairment in middle-aged and older adults with obstructive sleep apnoea: does excessive daytime sleepiness play a role?
}

\author{
To the Editor:
}

We read with great interest the original article entitled "Detection of mild cognitive impairment in middle-aged and older adults with obstructive sleep apnoea" [1], in which, the authors addressed the ability of two cognitive screening tests, the Mini-Mental State Examination (MMSE) and Montreal Cognitive Assessment (MoCA), to detect mild cognitive impairment (MCI) in obstructive sleep apnoea (OSA) patients. In this study, the authors conclude that in patients with OSA MoCA is an effective screening tool for cognitive impairment, while MMSE cannot acceptably detect MCI. Cognitive deficits in OSA is a very interesting topic, which has been not adequately studied and remains to be fully elucidated [2].

Recent studies have reported that excessive daytime sleepiness (EDS), a cardinal (but not pervasive) symptom of OSA, can be used as an indicator for identifying high-risk OSA patients. Indeed, an OSA patient with EDS represents a distinct clinical phenotype, since in numerous studies EDS has modified the relationship between severity of the disease (expressed by the apnoea-hypopnoea index) and incidence of arterial hypertension [3], glucose metabolism [4], healthcare utilisation [5] and mortality [6].

EDS may also contribute to neurocognitive impairment in OSA patients [7]. The presence of EDS in OSA has been found to be strictly related to sleep deprivation and fragmentation, which specifically lead to impaired attention and memory [7]. Therefore, it is conceivable that an increased percentage of amnestic MCI or specific multi-domain MCI (memory plus attention/executive function) can be found among OSA patients with EDS. Furthermore, regarding OSA treatment, limited data supports that continuous positive airway pressure fails to ameliorate cognition in OSA patients without EDS [7].

It would be of great interest if the authors would assess the role of EDS in their study. For example, if the presence of EDS modifies the discriminatory abilities of the MoCA and the MMSE in OSA patients as well as in controls, and/or to explore differences in the prevalence of MCI (and its subtypes) between sleepy and nonsleepy controls and patients with OSA.

We believe that if EDS occurrence is found to enhance the discriminatory abilities of these two tests, this can have important clinical implications and may facilitate an individualised diagnostic and therapeutic work-up of OSA patients, which remains a challenge [8], especially in the field of cognitive dysfunction in OSA.

Since the awareness of cognitive dysfunction in OSA is not very high [9], the identification and the evaluation of new screening tools for MCI in this clinical setting is crucial. Several other brief screening instruments to detect cognitive dysfunction in OSA patients could also be studied [10]. In addition, studies to prospectively explore the effect of this screening on important patients', caregivers' and clinicians' decision-making outcomes in OSA, are needed.

@ERSpublications

Excessive daytime sleepiness may modify the discriminatory abilities of the Mini-Mental State Examination and Montreal Cognitive Assessment tests to detect mild cognitive impairment in patients with obstructive sleep apnoea http://ow.ly/AFXx30mw9dm

Cite this article as: Steiropoulos P, Galbiati A, Ferini-Strambi L. Detection of mild cognitive impairment in middle-aged and older adults with obstructive sleep apnoea: does excessive daytime sleepiness play a role? Eur Respir J 2019; 53: 1801917 [https://doi.org/10.1183/13993003.01917-2018]. 
Paschalis Steiropoulos ${ }^{1,2}$, Andrea Galbiati ${ }^{1}$ and Luigi Ferini-Strambi ${ }^{1}$

${ }^{1}$ Dept of Neurology OSR-Turro-Sleep Disorders Center, Università Vita-Salute San Raffaele, Milan, Italy. ${ }^{2}$ Sleep Unit, Dept of Pneumonology, Medical School, Democritus University of Thrace, Alexandroupolis, Greece.

Correspondence: Paschalis Steiropoulos, Dept of Pneumonology, Medical School, Democritus University of Thrace, 68100 Alexandroupolis, Greece. E-mail: pstirop@med.duth.gr

Received: Oct 062018 | Accepted: Oct 192018

Conflict of interest: None declared.

\section{References}

1 Gagnon K, Baril A-A, Montplaisir J, et al. Detection of mild cognitive impairment in middle-aged and older adults with obstructive sleep apnoea. Eur Respir J 2018; 52: 1801137.

2 Leng Y, McEvoy CT, Allen IE, et al. Association of sleep-disordered breathing with cognitive function and risk of cognitive impairment: a systematic review meta-analysis. JAMA Neurol 2017; 74: 1237-1245.

3 Ren $\mathrm{R}$, Li Y, Zhang J, et al. Obstructive sleep apnea with objective daytime sleepiness is associated with hypertension. Hypertension 2016; 68: 1264-1270.

4 Nena E, Steiropoulos P, Papanas N, et al. Sleepiness as a marker of glucose deregulation in obstructive sleep apnea. Sleep Breath 2012; 16: 181-186.

5 Ronksley PE, Hemmelgarn BR, Heitman SJ, et al. Excessive daytime sleepiness is associated with increased healthcare utilization among patients referred for assessment of OSA. Sleep 2011; 34: 363-370.

6 Gooneratne NS, Richards KC, Joffe M, et al. Sleep disordered breathing with excessive daytime sleepiness is a risk factor for mortality in older adults. Sleep 2011; 34: 435-442.

7 Zhou J, Camacho M, Tang X, et al. A review of neurocognitive function and obstructive sleep apnea with or without daytime sleepiness. Sleep Med 2016; 23: 99-108.

8 Randerath W, Bassetti CL, Bonsignore MR, et al. Challenges and perspectives in obstructive sleep apnoea: Report by an ad hoc working group of the Sleep Disordered Breathing Group of the European Respiratory Society and the European Sleep Research Society. Eur Respir J 2018; 52: 1702616.

9 Bassetti CL, Ferini-Strambi L, Brown S, et al. Neurology and psychiatry: waking up to opportunities of sleep: State of the art and clinical/research priorities for the next decade. Eur J Neurol 2015; 22: 1337-1354.

10 Lin JS, O'Connor E, Rossom R, et al. Screening for Cognitive Impairment in Older Adults: An Evidence Update for the U.S. Preventive Services Task Force. Evidence Report No. 107. AHRQ Publication No. 14-05198-EF-1. Rockville, Agency for Healthcare Research and Quality, 2013.

Copyright @ERS 2019

\section{From the author:}

How sleepiness can inform clinicians about the presence of cognitive deficits and the risk of further cognitive decline in older adults is an intriguing question. The comments made by P. Steiropoulos and co-workers in their correspondence led us to perform supplementary analyses using the Epworth Sleepiness Scale in our cohort [1]. We first explored whether the presence of self-reported sleepiness modified the prevalence of mild cognitive impairment (MCI) in subjects with obstructive sleep apnoea (OSA) (mild, moderate and severe OSA were combined) and controls. In the OSA group, MCI tended to be less prevalent in subjects reporting sleepiness (Epworth Sleepiness Scale score of 10 and higher) compared to those reporting no sleepiness. More specifically, MCI was found in $31.7 \%$ of sleepy subjects and in $45.6 \%$ of nonsleepy OSA participants $\left(\chi^{2}(1)=2.05 ; \mathrm{p}=0.15\right)$. In the control group, sleepiness was not associated with a higher prevalence of MCI (37.5\% had MCI in nonsleepy controls compared to $33.3 \%$ with MCI in sleepy control subjects; $\left.\chi^{2}(1)=0.03 ; \mathrm{p}=0.86\right)$. When the association between sleepiness and MCI subtypes was explored (i.e. amnestic versus non-amnestic and single cognitive domain affected versus multiple cognitive domains affected), no group differences were found between OSA subjects with and without sleepiness (figure 1). Our exploratory results suggest that self-reported daytime sleepiness is not associated with increased neurocognitive impairments in our cohort of OSA and control participants.

@ERSpublications

Sleepiness does not affect the prevalence and subtype of mild cognitive impairment, and discriminative ability of cognitive screening tests in detecting mild cognitive impairment http://ow.ly/se9u30mMtYc

Cite this article as: Gagnon K, Gosselin N. Detection of mild cognitive impairment in middle-aged and older adults with obstructive sleep apnoea: does excessive daytime sleepiness play a role? Eur Respir J 2019; 53: 1802113 [https://doi.org/10.1183/13993003.02113-2018]. 
a)

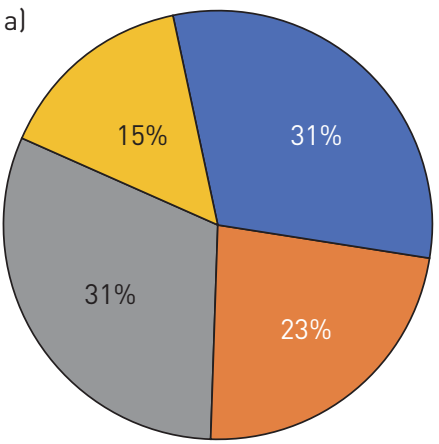

b)

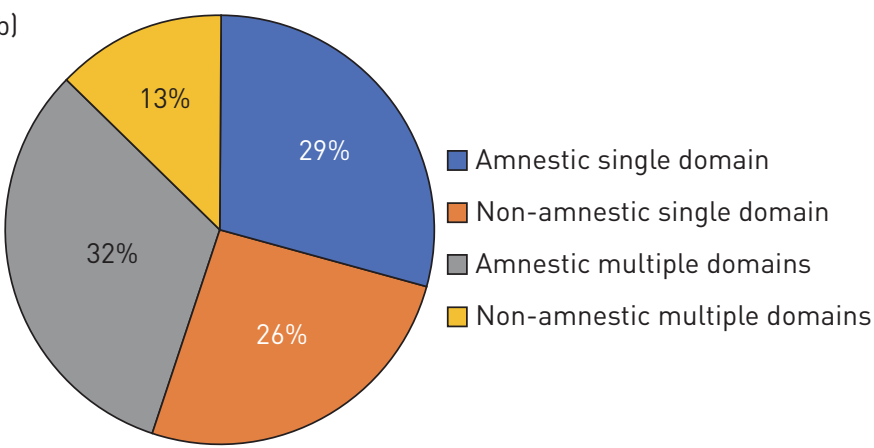

FIGURE 1 Proportions of mild cognitive impairment subtypes in a) obstructive sleep apnoea (OSA) participants with sleepiness and b) OSA participants without sleepiness. We observed a similar proportion of amnestic single domain between OSA with sleepiness (31\%) and OSA without sleepiness (29\%). The proportion of non-amnestic single domain was similar in OSA with (23\%) and without sleepiness (26\%). A similar proportion of amnestic multiple domains was found in OSA with (31\%) and without sleepiness (32\%). Both 0SA groups were similar in terms of the proportion of non-amnestic multiple domains subtype $(15 \%$ and $13 \%$ for sleepiness and no sleepiness, respectively).

We then explored whether the presence of self-reported sleepiness modified the discriminatory abilities of the Montreal Cognitive Assessment (MoCA) and the Mini-Mental State Examination (MMSE) in our OSA and control subjects. In the sleepy OSA group, the MoCA showed an area under the curve (AUC) of 0.82 (95\% CI 0.69-0.95) with an optimal cut-off value of 27, while the MMSE had an AUC of 0.67 (95\% CI $0.50-0.85$ ) and an optimal cut-off of 29. Similar results were observed in the OSA group without sleepiness: the MoCA showed an AUC of 0.84 (95\% CI 0.75-0.93; optimal cut-off of 27) whereas the MMSE had an AUC of 0.71 (95\% CI 0.58-0.83; optimal cut-off of 29). We were not able to perform the receiver operating characteristic curve analyses in the control group, because sleepiness was present in only six participants, with only two presenting a MCI. However, our exploratory analyses suggest that sleepiness in OSA participants does not affect the discriminative abilities of both cognitive screening tests in detecting MCI.

In conclusion, in our cohort of OSA and control participants, sleepiness was not associated with increased prevalence of MCI and/or its different subtypes. Sleepiness was also not associated with changes in MoCA and MMSE discriminatory abilities. Previous studies have shown that OSA with significant sleepiness could lead to reduced performance in cognitive tests measuring attention and memory [2]. Our OSA subjects are probably different from subjects included in some previous studies, as $40.4 \%$ of them had cognitive impairment severe enough to warrant a diagnosis of MCI. Cognitive impairment in our cohort may have caused difficulties in accurately assessing sleepiness using the Epworth Sleepiness Scale. In fact, we recently found that OSA participants with MCI report less subjective cognitive complaints compared to those without MCI, possibly because they had early impairment of metacognitive processes, including lower self-criticism and judgment [3]. Further studies should therefore use a combination of subjective and objective measures of sleepiness to understand its impact on cognitive functioning in older adults with OSA. How sleepiness could represent a marker of a more impacted brain in the context of OSA in ageing remains unclear. This question is relevant, since sleepiness is not only one of the first daytime consequences reported by OSA patients, but also a well-known behavioural symptom of early dementia [4].

Katia Gagnon ${ }^{1,2}$ and Nadia Gosselin ${ }^{1,3}$

${ }^{1}$ Center for Advanced Research in Sleep Medicine, Hôpital du Sacré-Cœur de Montréal, Montréal, QC, Canada. ${ }^{2}$ Dept of Psychology, Université du Québec à Montréal, Montréal, QC, Canada. ${ }^{3}$ Dept of Psychology, Université de Montréal, Montreal, QC, Canada.

Correspondence: Nadia Gosselin, Center for Advanced Research in Sleep Medicine, Hôpital du Sacré-Cœur de Montréal, 5400 boul. Gouin Ouest, Room J-5135, Montréal, Québec, H4J 1C5, Canada.

E-mail: nadia.gosselin@umontreal.ca

Received: Nov 062018 | Accepted: Nov 082018

Conflict of interest: K. Gagnon has nothing to disclose. N. Gosselin reports grants from Canadian Institutes of Health Research (government agency grant) and Fonds de Recherche en Santé du Québec (government agency grant and salary award), during the conduct of the study.

Support statement: This work was supported by the Government of Canada, Canadian Institutes of Health Research (MOP123294). Funding information for this article has been deposited with the Crossref Funder Registry. 


\section{References}

1 Gagnon K, Baril A-A, Montplaisir J, et al. Detection of mild cognitive impairment in middle-aged and older adults with obstructive sleep apnoea. Eur Respir J 2018; 52: 1801137.

2 Gagnon K, Baril A-A, Gagnon J-F, et al. Cognitive impairment in obstructive sleep apnea. Pathol Biol (Paris) 2014; 26: $233-240$.

3 Gagnon K, Baril A-A, Montplaisir J, et al. Subjective cognitive complaint in late middle-aged and older individuals with obstructive sleep apnea. Sleep 2017; 40: A104.

4 Rongve A, Boeve BF, Aarsland D. Frequency and correlates of caregiver-reported sleep disturbance in a sample of persons with early dementia. J Am Geriatr Soc 2010; 58: 480-486.

Copyright (CERS 2019 